\title{
EL PROCESO DE TOMA DE DECISIONES ESTRATÉGICAS EN LAS UNIVERSIDADES PÚBLICAS
}

\section{Introducción}

La sociedad que adviene se denomina "sociedad del conocimiento" y se caracteriza porque tanto el cambio en el entorno como la generación de nuevos conocimientos constituyen un proceso permanente y sistemático.

La fuente de la ventaja competitiva en la sociedad actual está en el conocimiento. Por esta razón las naciones, las organizaciones y las personas pueden lograr ventajas competitivas sustentables a través del conocimiento.

Sin embargo, existe una brecha significativa en el mundo respecto de cómo enfrentar y participar de esta nueva sociedad. Por ejemplo, América Latina representa alrededor del 8\% de la población mundial; pero, en términos relativos, tiene un déficit de profesionales que trabajan en investigación y desarrollo, en equipamiento tecnológico y en infraestructuras de comunicación. Más aún, tal como sostiene Brunner (Brunner, 2000), el conocimiento científico generado en conjunto por toda América Latina es inferior a los niveles que logran países como España o Suiza.

Por su parte, el nivel de escolaridad o años de estudios de la población en nuestra región es poco competitivo y por debajo de países a los que superábamos en décadas pasadas, como Malasia, Filipinas o Tailandia.

En Chile, el promedio de años de estudio es moderado pero creciente, aunque las pruebas internaciones del tipo Trends in International Mathematics and Science Study (TIMSS) muestran 
rendimientos deficientes, apuntando a una baja calidad de la educación. La proporción de profesionales respecto de la fuerza de trabajo es insuficiente considerando el nivel de desarrollo del país.

La inversión en investigación y desarrollo en Chile es escasa, comparada con los países de la Organisation for Economic Cooperation and Development (OECD) o, incluso, países emergentes como Brasil. El número de científicos y de patentes generadas, y el impacto del trabajo científico realizado, muestran a Chile como un país que requiere crecer mucho en estas dimensiones.

Las universidades tienen un papel fundamental impidiendo que estas brechas se sigan incrementando. La producción de conocimiento y su posterior difusión son sus tareas esenciales. Para ello se requiere un sistema robusto, con instituciones sanas y en crecimiento.

Ahora bien, dado que las instituciones universitarias -tanto públicas como privadas- tienen niveles importantes de autonomía y autogestión, que logren excelencia en su quehacer es un desafío que puede tener orientaciones a partir de las políticas públicas, pero que, irremediablemente, depende de cada una de las universidades.

Las universidades públicas que operan en un mismo sector industrial, que tienen una constitución jurídica y desafíos equivalentes, entre otras similitudes, pueden y suelen tener distintos resultados. De hecho algunas son más exitosas que otras. Estas diferencias se explican de manera significativa por la calidad de las decisiones estratégicas adoptadas.

Este trabajo entrega un conjunto de propuestas prácticas para llevar a cabo el proceso de diseño e implantación de las decisiones estratégicas en las universidades públicas.

\section{Rasgos idiosincrásicos}

Las universidades públicas chilenas creadas por ley forman parte del Consejo de Rectores y tienen una serie de rasgos distintivos 
que determinan e influyen significativamente en el diseño y la implantación de sus procesos estratégicos, los que se detallan a continuación (Rodríguez, 2005):

- Generación de bienes públicos: Las universidades públicas generan bienes privados, pero también una proporción significativa de bienes públicos. Ponen al alcance de la ciudadanía, en las diversas regiones del país, el acceso equitativo a una educación de calidad. Las universidades públicas, como red de instituciones, garantizan que, a lo largo del país, existirá al menos una universidad de calidad en cada región, que tendrá como prioridad el acceso de los más capaces, con independencia de su credo, su condición socioeconómica, etc.

Tan importante como lo anterior es que las universidades públicas garantizan la diversidad y el pluralismo en cada región. Es decir, en cada una ingresan los mejores postulantes y allí aprenden a compartir, a conocerse, a integrarse, basados en sus talentos y en sus capacidades, y no en su situación social ni económica.

Estas instituciones generan la mayor proporción de la ciencia y la tecnología en Chile. Son el referente obligado para medir las publicaciones relevantes producidas en el país. Además, se vinculan permanentemente con sus comunidades regionales y son un punto de desarrollo social, cultural y humano.

- Sistema de elección de autoridades: En las universidades públicas, la elección del rector, decanos, directores de departamentos, escuelas o institutos se efectúa habitualmente por parte de sus pares académicos. Por lo tanto, las autoridades universitarias tienen una condición transitoria y, en esencia, se constituyen en representantes de sus electores.

La evidencia muestra que en Chile es común la reelección de autoridades en el sistema público. Esta situación implica al menos dos consideraciones: primero, a las autoridades no les 
desagrada su condición transitoria y les parece atractivo mantener dicha condición por un tiempo mayor al de la elección inicial; segundo, una elección y una posterior reelección supone una base de apoyo entre sus pares y una correcta representación de los intereses corporativos e individuales. No obstante, estos no siempre están correlacionados ni en perfecta armonía.

La elección de autoridades por sus pares tiene algunos riesgos desde la perspectiva de la dirección estratégica, ya que el diseño y la implantación de las habilidades se puede ver influenciada por aspectos políticos y por intereses privados no necesariamente coligados con los intereses corporativos.

- Ausencia de un criterio orientador único: ¿Cómo se mide y quién mide el éxito de una universidad pública? ¿Cómo se evalúa el éxito de la gestión de un determinado rector y de su equipo directivo?

Las respuestas no son triviales y aluden a un aspecto central de estas casas de estudio. No existe un criterio único de éxito ni un fin claro y exclusivo que perseguir. Tampoco un único evaluador del desempeño de las universidades públicas.

Al asignar el aporte fiscal directo concursable o al definir los aranceles de referencia, el Ministerio de Educación entrega una noción del éxito relativo de las universidades, de acuerdo con el número e índice de impacto de las publicaciones reconocidas por el Institute for Scientific Information (ISI) o por Scientific Electronic Library Online (SciELO), por el número e impacto de los proyectos del Fondo Nacional de Desarrollo Científico y Tecnológico (FONDECYT) o del Fondo de Fomento al Desarrollo Científico y Tecnológico (FONDEF). También, por el índice de deserción de estudiantes, la duración de las carreras, el número de doctores y magíster por estudiante, entre otras variables.

Para ciertos académicos algunos de estos indicadores serán irrelevantes; incluso, más de alguno planteará que esta es una mirada 
reduccionista del quehacer universitario. En cualquier caso, ya sea que acepten o no los indicadores anteriores, para ellos su nivel de satisfacción personal será una variable clave para evaluar el éxito de una gestión o de su universidad.

Los funcionarios no académicos verán en sus remuneraciones, en las prestaciones sociales y de bienestar, y en la estabilidad y carrera funcionaria los criterios básicos para medir cuán bien está la universidad o cuán buena ha sido una determinada gestión.

Los estudiantes, en cambio, pensarán en el prestigio de la institución, en la tasa de colocación de los egresados, en la remuneración promedio de los egresados, en los índices de deserción y duración de las carreras, en la infraestructura, el equipamiento y la calidad de los docentes.

Se puede seguir sumando miradas, de las autoridades centrales y regionales, de los parlamentarios, de los empresarios, de la comunidad en general. La cuestión central es que en las universidades públicas no existen medidas generalmente aceptadas de éxito, y los agentes que tienen derecho a evaluar a estas instituciones son variados y con intereses múltiples. En consecuencia, el norte estratégico de una institución universitaria no surge en forma espontánea; este es un punto de discusión de la comunidad y de los distintos actores que inciden en las decisiones estratégicas en las universidades públicas.

- Múltiples influencias internas y externas: Al interior de la universidad, los académicos, no académicos y estudiantes tienen una influencia decisiva sobre el curso estratégico de las instituciones públicas. Los gobiernos regionales, las autoridades provinciales y locales, los colegios del entorno específico, las autoridades del gobierno central y la comunidad en general tienen también opinión y algún grado de influencia sobre el accionar de las universidades públicas. 
- Múltiples fuentes de financiamiento: Las estrategias de desarrollo requieren recursos para su implantación. Es necesario destacar que las universidades públicas tienen un componente de financiamiento estatal y otro de financiamiento privado. A partir de 1981, las políticas de educación superior han conducido a que las universidades públicas dependan cada vez más del mercado y de los recursos captados a través de aranceles de matrícula de pregrado, aranceles de posgrado, venta de servicios, consecución de recursos para investigación, donaciones, entre otros.

Por lo demás, los recursos públicos han sido, en general, distribuidos a partir de concursos entre las universidades del Consejo de Rectores. La búsqueda de estudiantes con altos puntajes de ingreso, la formación de doctores y magíster, la generación de publicaciones ISI y, más recientemente, SciELO, la consecución de proyectos FONDECYT y FONDEF, son algunas de las orientaciones que han guiado la asignación de recursos públicos al sistema universitario chileno.

En consecuencia, el análisis de los aspectos estratégicos en una universidad pública debe considerar estos rasgos distintivos, es decir, que las universidades públicas generen bienes públicos, elijan a sus autoridades, tengan múltiples influencias internas y externas, y múltiples fuentes de financiamiento.

\section{La dirección universitaria y la toma de decisiones estratégicas}

La fuente de la ventaja competitiva en la sociedad del conocimiento está en el acto de compartir y crear conocimiento, y esto se logra mediante la toma de decisiones estratégicas (Kim \& Maugborne, 1998; Cool, 1998).

La dirección estratégica ha generado un conjunto de modelos, generalmente aceptados, que indican los pasos que se deben seguir 
para diseñar las estrategias corporativas y competitivas. Sin embargo, cuando se trata de dilucidar cómo seguir esos pasos, es decir, cómo tomar las decisiones estratégicas, allí la literatura es menos clara.

Recientemente, Brunner (Brunner, 2005), discutiendo acerca de cómo pueden gestionarse las universidades, recordó que estas han sido gobernadas por Papas, príncipes, profesores e, incluso, por estudiantes, por sus propietarios o por los mandantes de la academia. Los modelos de dirección utilizados han sido variados: autocráticos, democráticos, burocráticos, políticos, entre otros. Sin embargo, en todos los casos se han debido compatibilizar las tradiciones autonomistas e individualistas de los académicos con los requisitos de conducción y coordinación necesarios para subsistir como una organización. Hoy la balanza del gobierno universitario se inclina inevitablemente hacia el polo de la gestión. En todo caso, según Brunner, no se trata de una gestión cualquiera: gobernar bien una universidad consiste en dotarla de los medios que la hagan capaz de liderar y gestionar el cambio, sin sacrificar las tradiciones académicas.

Sin duda, esta contribución intelectual es una exquisita oportunidad para exponer una propuesta acerca de cómo emprender el gobierno universitario en el mundo real.

En efecto, la expresión concreta de los procesos de dirección estratégica está en la toma de decisiones: allí está la fuente de la ventaja competitiva. Las decisiones estratégicas son elecciones importantes, no rutinarias, que implican una cantidad significativa de recursos y cuyos efectos son de largo alcance.

En un entorno similar, es decir, dentro de un mismo sector industrial, la diferencia entre las organizaciones que tendrán éxito o fracaso estará en la calidad de sus decisiones.

Las ideas que se exponen a continuación resultan de un conjunto de investigaciones en instituciones universitarias de Chile, 
Perú y España (Rodríguez, 2005; Rodríguez, 2004), y de la experiencia del autor en cargos de alta dirección universitaria.

\section{Imperativos estratégicos para la toma de decisiones en las instituciones universitarias}

Las instituciones universitarias, siguiendo la clasificación de Mintzberg (Mintzberg, 1979), se caracterizan por operar en un ambiente estable, complejo y con un nivel creciente de hostilidad. Éstas no tienen un criterio orientador único, tienen múltiples fuentes de ingresos y se ven enfrentadas a importantes influencias internas y externas.

Dado este contexto interno y externo, surge una serie de imperativos estratégicos para lograr éxito en la formulación e implantación de las decisiones y, por ende, contribuir al éxito de las universidades. Es necesario:

\subsection{Fomentar la racionalidad}

La racionalidad se define como el grado en el cual el proceso de decisiones refleja un deseo de tomar la mejor decisión para alcanzar los objetivos bajo las circunstancias dadas (Simon, 1980). Trata de recoger la información necesaria para formar expectativas acerca de ciertas variables, realiza una búsqueda profunda de información y la emplea de modo exhaustivo en la adopción de la decisión final. Cuanto más racional sea la decisión más importantes serán las técnicas cuantitativas, y el criterio analítico tendrá supremacía sobre la intuición (Dean \& Sharfman, 1993).

Es necesario lograr un alto nivel de racionalidad en el proceso de toma de decisiones para conseguir una buena calidad en la formulación o diseño de la decisión. Para adoptar una decisión racional es necesario:

- Buscar en forma intensiva la información relevante para la generación de opciones y alternativas a la decisión. 
- Realizar un análisis riguroso de la información relevante para lograr una evaluación adecuada y exhaustiva de las opciones de la decisión.

- Adoptar la decisión basándose en un análisis crítico y exhaustivo, buscando sustento en el criterio analítico más que en la intuición.

\subsection{Reducir el nivel de politización}

La politización indica el grado en el cual la toma de decisiones obedece a razones de carácter político o a influencias de grupos de intereses o a coaliciones que se forman para favorecer sus propias preferencias (Papadakis, Lioukas \& Chambers, 1998).

La politización en el proceso de toma de decisiones tiene efectos negativos sobre la implementación de la decisión. Para reducirla es necesario minimizar:

- El impacto de grupos o coaliciones con intereses diferentes.

- Las negociaciones entre los grupos de interés.

- El empleo de tácticas tales como el "lobby", el manejo de agenda, el ocultamiento de información, entre otras.

- La ruptura de las conversaciones durante el proceso de adopción de la decisión.

\subsection{Fomentar el conflicto cognitivo}

El conflicto cognitivo es el grado en el cual existen intereses o posiciones cognitivas encontradas o contrapuestas entre quienes adoptan las decisiones (Amason, 1996). Tiene efectos positivos sobre la racionalidad, la cual, a su vez, influye sobre la calidad de la formulación de las decisiones estratégicas. Para estimularlo es necesario:

- Discutir la decisión desde diferentes enfoques o perspectivas.

- Favorecer la discusión de ideas diferentes.

- Estimular el intercambio de ideas y opiniones entre los participantes. 


\subsection{Reducir el conflicto afectivo}

Este conflicto se asocia con la existencia de fricciones, choques de personalidad, tensión y fuertes discusiones durante la toma de decisiones (Amason, 1996). Influye positivamente sobre la politización, variable que tiene efectos negativos sobre la implantación de las decisiones estratégicas. Para minimizarlo es necesario:

- Reducir las fricciones y evitar los choques de personalidad entre los participantes.

- Reducir la tensión del ambiente en el proceso decisional.

\subsection{Fomentar la flexibilidad}

La flexibilidad es el grado en el cual las personas encargadas de tomar las decisiones exploran nuevas ideas o supuestos en este proceso (Dean \& Sharfman, 1996). Tiene efectos directos sobre la racionalidad, variable que influye sobre la calidad de la decisión adoptada. Para lograr flexibilidad en la toma de decisiones es necesario:

- Confiar en nuevas fuentes de información.

- Recibir en forma positiva las nuevas ideas y analizarlas rigurosamente.

- Lograr que los participantes contribuyan en forma amplia y creativa, más allá de las materias definidas por su cargo.

- Reconsiderar las posiciones originales.

\subsection{Fomentar la justicia procesal}

La justicia procesal se asocia con el grado en el cual los procesos dinámicos de decisión son juzgados como correctos por quienes participan en dichos procesos (Kim \& Maugborne, 1991; (Kim \& Maugborne, 1993). Tiene efectos positivos sobre la implantación de las decisiones estratégicas; en consecuencia, es deseable favorecer un proceso justo en la adopción de las decisiones. Para fortalecer la justicia procesal es necesario:

- Dar a los participantes la posibilidad de refutar las ideas de otros. 
- Considerar la opinión de los participantes del proceso decisional.

- Explicar las razones por las cuales unas ideas prevalecen sobre otras en la decisión final.

- Dar a conocer a los participantes lo que se espera de ellos antes, durante y después de la decisión.

Para favorecer el desarrollo de un proceso exitoso que tienda a la búsqueda de la racionalidad, a minimizar la politización y el conflicto afectivo, pero que fomente el conflicto cognitivo, la flexibilidad y la justicia procesal en el proceso de toma de decisiones estratégicas en las instituciones universitarias se requieren los restantes tres factores.

\subsection{Fomentar la diversidad funcional y de conocimientos}

La pluralidad de estudios forma parte de la diversidad funcional y de conocimientos y se refiere al grado en el cual existen diferencias en el nivel de formación y preparación formal de los participantes en el proceso de toma de decisiones estratégicas. Esta diversidad contribuye al conflicto cognitivo, el que, a su vez, es una variable que explica la racionalidad de este proceso. Desde esta visión, es importante fomentar la participación de personas:

- Que ocupan diferentes puestos en la organización.

- Que representan diferentes especialidades o áreas de desarrollo en la organización.

- Con diferentes niveles de estudios formales en el proceso de toma de decisiones.

4.8. Propender hacia la búsqueda de la congruencia de valores entre los participantes en el proceso

La congruencia de valores se refiere al grado en que los valores, creencias y metas de los miembros del equipo de toma de decisiones son similares. Es una variable fundamental para reducir 
el conflicto afectivo y la politización en este proceso. En este sentido es relevante que:

- Los miembros del equipo de alta dirección compartan los valores esenciales y tengan una concepción valórica dominante, de modo tal que este equipo sea concebido como una unidad.

- Los miembros que participen en la decisión tengan creencias similares respecto del desarrollo de la universidad y estén de acuerdo con las prioridades estratégicas de la universidad.

\subsection{Fomentar un estilo de liderazgo participativo y colaborativo}

El estilo de liderazgo participativo se refiere al grado en el cual el líder del proceso de toma de decisiones considera la opinión, el parecer, la posición, las sugerencias y los consejos de los participantes en el proceso decisional. Este estilo tiene efectos sobre la flexibilidad del proceso de toma de decisiones y la justicia procesal, aspectos que, a su vez, influyen sobre la racionalidad y, por ende, sobre la formulación de la decisión y, directamente, sobre la implantación de la decisión. En virtud de lo anterior es importante que el líder:

- Considere la opinión de los participantes.

- Pregunte el parecer a los participantes.

- Pondere las posiciones de los participantes.

- Incorpore al debate las contribuciones de los participantes.

- Esté dispuesto a aceptar consejos y a emplear dichos consejos.

- Muestre un afán de colaboración permanente frente a los participantes.

- Considere el bienestar de los participantes durante el proceso decisional.

- Trate como iguales a los miembros del grupo.

A partir del análisis anterior, es posible bosquejar un modelo de los imperativos estratégicos para lograr éxito en la toma de decisiones como el de la figura 1: 


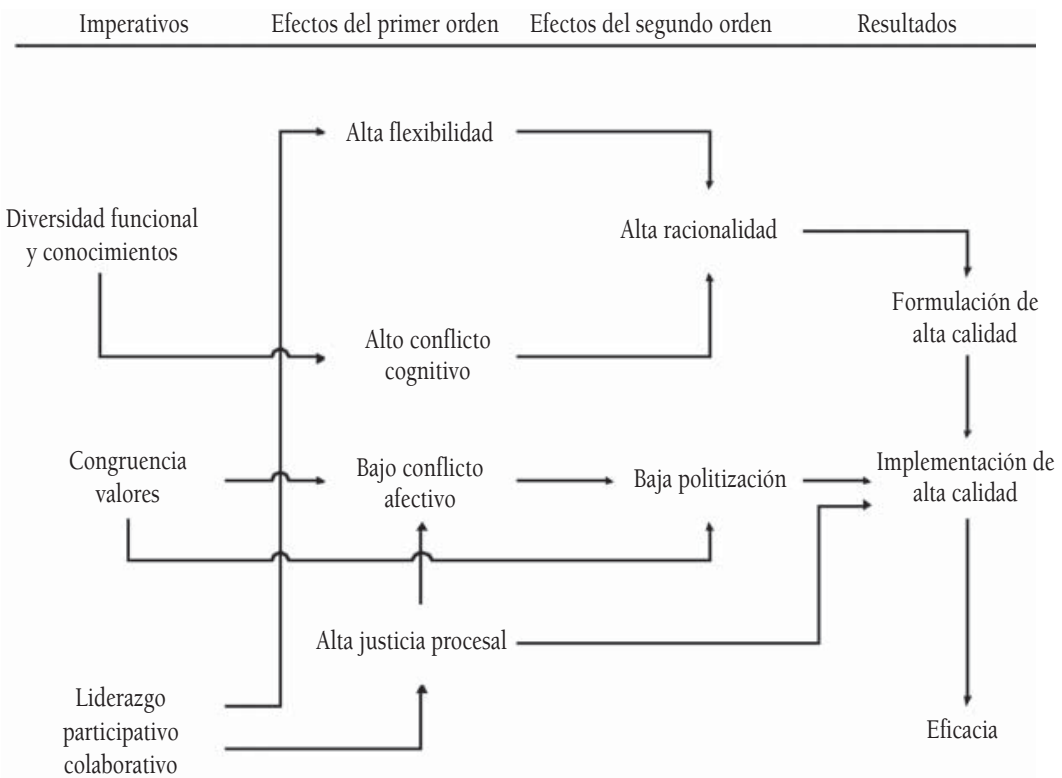

Figura 1: Imperativos estratégicos para la toma de decisiones en las universidades de España y Chile.

\section{Conclusiones}

En las universidades públicas no existe un único criterio para medir el éxito; sin embargo, la evidencia muestra que la calidad de diseño de las decisiones estratégicas y su posterior implantación marcan la diferencia entre este tipo de instituciones.

Las universidades públicas que generan mejores decisiones lograrán una mejor posición competitiva y, por tanto, tendrán una ventaja sobre otras organizaciones que operan en el mismo sector industrial.

La calidad de la formulación de las decisiones depende del grado de racionalidad logrado en el proceso. Por su parte, la racionalidad se explica por la flexibilidad y el conflicto cognitivo con que se diseña la decisión. 
La implementación de las decisiones estratégicas se explica negativamente con el grado de politización del proceso decisional, el que depende del conflicto afectivo y se ve reducido por la justicia procesal percibida por los participantes del proceso.

En este contexto, las características del equipo de alta dirección -tales como la diversidad, la congruencia de valores y el estilo de liderazgo- son las variables determinantes del proceso de toma de decisiones y de sus resultados.

En consecuencia, contar con un determinado equipo directivo $u$ otro en las universidades públicas no sólo variará los énfasis o los estilos, sino que será decisivo para el desarrollo de largo plazo de las instituciones.

\section{Referencias bibliográficas}

Amason, A. C. (1996) Distinguishing the effects of functional and dysfunctional conflict on strategic decision making: Resolving a paradox for top management teams. Academy of Management Journal, 39, (1), pp.123-148.

Brunner, José J. (2000) Educación superior: Desafío y tareas. Discurso de incorporación a la Academia Chilena de Ciencias Sociales, Políticas y Morales.

Brunner, José J. (2005) Gobierno Universitario. El Mercurio, 22 de marzo.

Cool, K. (1998) Commentary on procedural justice, strategic decision making, and the knowledge economy. Strategy Management Journal, 19, pp.339-341.

Dean, J \& Sharfman, M.(1993) The relationship between procedural rationality and political behavior in strategic decision making. Decision Science, 24, (6), pp. 1069-1083.

Dean, J. \& Sharfman, M. (1996) Does decision process matter? A study of strategic decision-making effectiveness. Academy of Management Journal, 39, (2), pp. 368-396.

Kim, W. \& Maugborne R. (1991) Implementing global strategies: The role of procedural justice. Strategic Management Journal, 12, pp. 125-143. 
Kim, W. \& Maugborne R. (1993). Procedural justice attitudes, and subsidiary top management compliance with multionational's corporate strategic decisions. Academy of Management Journal, 36, (3), pp. 125-143.

Kim, W. \& Maugborne R. (1998) Procedural justice, strategic decision making, and the knowledge economy. Strategic Management Journal, 19, pp. 323-338.

Mintzberg, Henry (1979) La estructuración de las organizaciones. New Jersey: Prentice Hall.

Papadakis, V.; Lioukas S. \& Chambers, D. (1998) Strategic decision-making proccesses: The role of management and context. Strategic Management Journal, 19, pp. 115-147.

Rodriguez, Emilio (2004) La toma de decisiones estratégicas: Una perspectiva integradora. Santiago de Chile: Ediciones Consorcio de Universidades Estatales.

Rodriguez, Emilio (2005) La toma de decisiones estratégicas en las instituciones universitarias: un estudio empírico en Chile y España. Santiago de Chile: Ediciones Consejo de Rectores.

Simon, Herbert (1980) Rationality as process and as product of thought. American Economic Review, 68, (2), pp. 1-16.

Recibido: 22 de mayo de 2006

Aceptado: 15 de junio de 2006 